\title{
A NONABELIAN TWO-DIMENSIONAL COHOMOLOGY FOR ASSOCIATIVE ALGEBRAS ${ }^{1}$
}

\author{
BY PAUL DEDECKER ${ }^{2}$ AND ABRAHAM S.-T. LUE \\ Communicated by O. G. Harrold, June 27, 1966
}

In a previous paper [4], a nonabelian cohomology theory for associative algebras was proposed for dimensions 0 and 1 . The purpose of this paper is to extend this theory to dimension 2. The methods used are closely analogous to those employed in the corresponding theory for groups (cf. [1],[2]). Throughout this paper all algebras will be associative algebras over some fixed commutative ring $\Lambda$ with identity. The term homomorphism without qualification will mean homomorphism of $\Lambda$-algebras.

1. One-cochains and one-cocycles. We consider algebras $B$ and $M$, and homomorphisms $\rho: B \rightarrow M, \Phi: M \rightarrow \mathfrak{M}(B)$, where $\mathfrak{T}(B)$ denotes the algebra of bimultiplications of $B$ (cf. [4],[5]). The system $B$ $=(B, M ; \rho, \Phi)$ is said to define the structure of an $M$-crossed module on $B$ if the following conditions are satisfied:

(i) the image of $\Phi$ is permutable on $B$;

(ii) $\rho(\Phi(m) b)=m \rho(b), \rho(b \Phi(m))=\rho(b) m$, for all $b \in B, m \in M$;

(iii) the composite $\Phi \rho: B \rightarrow \operatorname{Tr}(B)$ maps each element of $B$ onto the inner bimultiplication which it defines.

We recall that a subset $S$ of $\Re(B)$ is permutable on $B$ if $(\xi b) \eta=\xi(b \eta)$, for all $\xi, \eta \in S, b \in B$ (cf. [4],[5]). The $M$-crossed module $B$ is an $M$ bimodule under the action defined by $\Phi$, and the condition (ii) shows that $\rho$ is a homomorphism of $M$-bimodules. Moreover $\rho(B)$ is an ideal in $M$ and the quotient algebra $L=M / \rho B$ has a canonical map $\Psi: L \rightarrow \mathscr{N}(Z)$, deduced from $\Phi$, where $Z=\operatorname{Ker}(\rho)$ is called the center of the crossed module $B$. $M$ and $L$ will be called the operator and outer operator algebras of $B$ respectively.

Given $B=(B, M ; \rho, \Phi)$, we define a 1-cochain from an algebra $A$ to $B$ to be a pair $(p, \phi)$ of maps $p: A \rightarrow B, \phi: A \rightarrow M$ and denote the set of these 1 -cochains by $\mathbb{C}^{1}(A, B) \cdot \mathcal{Z}^{1}(A, \beta)$ is the subset consisting of the 1-cocycles, namely those pairs $(p, \phi)$ for which

$\phi$ is a homomorphism;

(1) $p$ is a homomorphism of $\Lambda$-modules;

$$
p\left(a_{1} a_{2}\right)=p\left(a_{1}\right) p\left(a_{2}\right)+p\left(a_{1}\right) \phi\left(a_{2}\right)+\phi\left(a_{1}\right) p\left(a_{2}\right), \quad a_{1}, a_{2} \in A .
$$

${ }^{1}$ Research partially supported by NATO research grant No. 224 .

2 Visiting Professor, Florida State University, Tallahassee, supported in part by NSF-G 5458. 
The composition $(q, \psi)+(p, \phi)$ in $\mathbb{e}^{1}(A, \beta)$ is defined only if $\psi=\rho p+\phi$, in which case

$$
(q, \psi)+(p, \phi)=(q+p, \phi) .
$$

$\mathbb{C}^{1}(A, \mathbb{B})$ together with this composition form a groupoid. From (1) we see that $\mathcal{Z}^{1}(A, \Theta)$ forms a subgroupoid of $\mathbb{C}^{1}(A, \beta)$.

2. Two-cochains and two-cocycles. From the algebra $A$, we define $F(A)$ to be the free $\Lambda$-module on the generators $\{\vec{a} \mid a \in A\}$. The map $\bar{a} \rightarrow a$ extends to an epimorphism $F(A) \rightarrow A$ of $\Lambda$-modules, with kernel $N(A)$. Hence $\sum_{i} \lambda_{i} \bar{a}_{i} \in N(A)$ for $\lambda_{i} \in \Lambda, a_{i} \in A$, if and only if $\sum_{i} \lambda_{i} a_{i}$ $=0$.

A 2-cochain from $A$ to $B$ is defined to be a triple $\left(\gamma_{1}, \gamma_{2}, \phi\right)$, of maps

$$
\gamma_{1}: A \times A \rightarrow B, \quad \gamma_{2}: N(A) \rightarrow B, \quad \phi: A \rightarrow M .
$$

A 2-cocycle is a 2-cochain which satisfies the conditions:

(I) $\phi\left(a_{1}\right) \gamma_{1}\left(a_{2}, a_{3}\right)-\gamma_{1}\left(a_{1} a_{2}, a_{3}\right)+\gamma_{1}\left(a_{1}, a_{2} a_{3}\right)-\gamma_{1}\left(a_{1}, a_{2}\right) \phi\left(a_{3}\right)=0$ ；

(II) $\sum_{i} \lambda_{i} \gamma_{1}\left(a, a_{i}\right)=\gamma_{2}\left(\sum_{i} \lambda_{i} \overline{a a_{i}}\right)-\phi(a) \gamma_{2}\left(\sum_{i} \lambda_{i} \bar{a}_{i}\right)$;

(III) $\sum_{i} \lambda_{i} \gamma_{1}\left(a_{i}, a\right)=\gamma_{2}\left(\sum_{i} \lambda_{i} \overline{a_{i} a}\right)-\gamma_{2}\left(\sum_{i} \lambda_{i} \bar{a}_{i}\right) \phi(a)$;

(IV) $\gamma_{2}$ is a homomorphism of $\Lambda$-modules;

(V) $\rho \gamma_{2}\left(\sum_{i} \lambda_{i} \tilde{a}_{i}\right)=\sum_{i} \lambda_{2} \phi\left(a_{i}\right)$;

(VI) $\rho \gamma_{1}\left(a_{1}, a_{2}\right)=\phi\left(a_{1} a_{2}\right)-\phi\left(a_{1}\right) \phi\left(a_{2}\right)$;

for $\lambda_{i} \in \Lambda, a, a_{1}, a_{2}, a_{3}, a_{i} \in A, \sum_{i} \lambda_{i} \bar{a}_{i} \in N(A)$.

$C^{2}(A, B)$ and $Z^{2}=Z^{2}(A, \Theta)$ will denote respectively the sets of 2cochains and 2-cocycles. Note that $(0,0, \phi) \in Z^{2}$ iff $\phi: A \rightarrow M$ is a homomorphism. These special 2-cocycles form a privileged subset $I^{2}=I^{2}(A, B)$ of $Z^{2}$, the elements of which are called neutral. Sometimes a special such $\phi$ is chosen corresponding to a base cocycle in $Z^{2}$; if $\phi$ is the zero morphism, then one speaks of the zero-cocycle.

Every extension $E \equiv 0 \rightarrow B \rightarrow^{\circ} E \rightarrow{ }^{\bullet} A \rightarrow 0$ of algebras gives rise to a commutative diagram with exact rows

$$
\begin{aligned}
& \begin{array}{cll}
0 \rightarrow B \stackrel{\iota}{\rightarrow} & E \stackrel{\epsilon}{\rightarrow} & A \rightarrow 0 \\
\| & \downarrow \Theta & \downarrow \theta
\end{array} \rightarrow \\
& B \rightarrow \operatorname{Ir}(B) \rightarrow \odot(B) \rightarrow 0
\end{aligned}
$$

where $\odot(B)$ denotes the algebra of outer bimultiplications (cf. [4], [5]), and the homomorphism $\Theta: E \rightarrow \operatorname{Tr}(B)$ is given by the multiplication in $E$. If further, $B$ is an $M$-crossed module, and we have a homomor- 
phism $\mu: E \rightarrow M$ such that $\Phi \mu=\Theta$, then the couple $(E, \mu)$ will be called an $M$-extension. We then have a commutative diagram with exact rows

$$
\begin{aligned}
& 0 \rightarrow B \stackrel{\iota}{\rightarrow} E \stackrel{\epsilon}{\rightarrow} A \rightarrow 0 \\
& 0 \rightarrow Z \rightarrow B \stackrel{\rho}{\rightarrow} M \rightarrow L \rightarrow 0
\end{aligned}
$$

and the homomorphism $\nu$ is called the crest of the $M$-extension. Two $M$-extensions $(E, \mu),\left(E^{\prime}, \mu^{\prime}\right)$ are equivalent if there is a morphism $E \rightarrow E^{\prime}$ of short exact sequences which extends the identity maps on $B$ and $A$, and for which the composite homomorphism $E \rightarrow E^{\prime} \rightarrow \mu^{\prime} M$ is precisely $\mu$. We denote by $\operatorname{Ext}(A, B)$ the set of equivalence classes of $M$-extensions. The crest $\nu$ in (4) corresponds to a map

$$
\kappa: \operatorname{Ext}(A, \beta) \rightarrow \operatorname{Hom}(A, L) .
$$

$(E, \mu)$ is called inessential if $E$ is cleft, i.e., if there exists a homomorphism of algebras $t: A \rightarrow E$ such that $\epsilon t=1_{A}$; it is called trivial if $E$ is the direct sum algebra of $B$ and $A$, and $\iota(b)=(b, 0), \epsilon(b, a)=a$, and if $\mu=0$.

3. Cohomology classes. It is now possible to define a map $\delta$ :

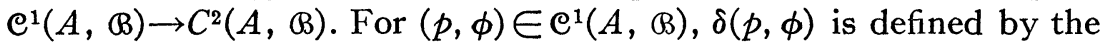
equation

$$
\delta(p, \phi)=\left(\delta_{1}(p, \phi), \delta_{2}(p, \phi), \phi\right),
$$

where, for $a_{1}, a_{2} \in A, \sum_{i} \lambda_{i} \bar{a}_{i} \in N(A)$,

$$
\delta_{1}(p, \phi)\left(a_{1}, a_{2}\right)=p\left(a_{1} a_{2}\right)-p\left(a_{1}\right) p\left(a_{2}\right)-p\left(a_{1}\right) \phi\left(a_{2}\right)-\phi\left(a_{1}\right) p\left(a_{2}\right) ;
$$

$$
\delta_{2}(p, \phi)\left(\sum_{i} \lambda_{i} \bar{a}_{i}\right)=\sum_{i} \lambda_{i} p\left(a_{i}\right) .
$$

For $(q, \rho p+\phi),(p, \phi) \in \mathfrak{C}^{1}(A, \beta)$, we obtain from (2) and (6)

$$
\begin{aligned}
& \delta_{1}\{(q, \rho p+\phi)+(p, \phi)\}=\delta_{1}(q, \rho p+\phi)+\delta_{1}(p, \phi), \\
& \delta_{2}\{(q, \rho p+\phi)+(p, \phi)\}=\delta_{2}(q, \rho p+\phi)+\delta_{2}(p, \phi) .
\end{aligned}
$$

Observe from (6) that $\delta(p, \phi)=(0,0, \phi)$ if and only if the last two conditions of (1) are satisfied. Hence, for $\phi$ a homomorphism, $\delta(p, \phi)$ $=(0,0, \phi)$ iff $(p, \phi) \in Z^{1}(A, \beta)$.

We now define an action $\Delta$ (on the left) of the groupoid $\mathfrak{e}^{1}(A, \mathbb{B})$ on the sets $C^{2}(A, ß)$ and $Z^{2}(A, ß)$. This is given by maps 


$$
\begin{aligned}
& \Delta: \mathfrak{C}^{1}(A, \beta) \times C^{2}(A, \beta) \rightarrow C^{2}(A, \beta), \\
& \Delta: \mathcal{C}^{1}(A, \beta) \times Z^{2}(A, \beta) \rightarrow Z^{2}(A, \beta) .
\end{aligned}
$$

For $(p, \phi) \in \mathfrak{e}^{1}(A, \beta),\left(\gamma_{1}, \gamma_{2}, \phi\right) \in C^{2}(A, \beta), \Delta\left\{(p, \phi) \times\left(\gamma_{1}, \gamma_{2}, \phi\right)\right\}$ is defined to be $\left(\gamma_{1}^{\prime}, \gamma_{2}^{\prime}, \phi^{\prime}\right)$, given by the equations

$$
\phi^{\prime}=\rho p+\phi, \quad \gamma_{1}^{\prime}=\delta_{1}(p, \phi)+\gamma_{1}, \quad \gamma_{2}^{\prime}=\delta_{2}(p, \phi)+\gamma_{2} .
$$

We easily verify that if $\left(\gamma_{1}, \gamma_{2}, \phi\right) \in Z^{2}(A, ß)$, then so does $\left(\gamma_{1}^{\prime}, \gamma_{2}^{\prime}, \phi^{\prime}\right)$. It follows from $(7)$ that, under $\Delta, \mathfrak{e}^{1}(A, B)$ is a groupoid of left operators.

The orbits in $Z^{2}(A, B)$ under $\Delta$ are called the thick 2-cohomology classes, and the set of these classes is denoted by $H^{2}(A, B)$. This is a set with preferred subset of neutral classes (i.e. containing a neutral cocycle) and with an eventual base or zero-class (containing the base or the zero 2-cocycle). There are also maps $k$ and $\kappa^{\prime}$ falling into the following commutative diagram with canonical horizontal arrow:

$$
\begin{gathered}
Z^{2}(A, B) \longrightarrow H^{2}(A, \beta) \\
k \backslash \searrow / \kappa^{\prime} \\
\operatorname{Hom}(A, L)
\end{gathered}
$$

and such that $k\left(\gamma_{1}, \gamma_{2}, \phi\right)=\pi \circ \phi$ with $\pi: M \rightarrow L$ canonical.

We see that both sets $\operatorname{Ext}(A, \beta)$ and $H^{2}(A, B)$ are provided with a privileged subset, an eventual base point and maps $\kappa$ and $\kappa^{\prime}$ as in (5) and (9). One can also show that an abelian group $H_{\nu}^{2}(A, Z)$ acts in a simply transitive way on the fibres $\kappa^{-1}(\nu)$ and $\kappa^{\prime-1}(\nu)$ in $\operatorname{Ext}(A, B)$ and $H^{2}(A, \Theta)$. Those sets therefore have spider structures in the sense of [6]. Moreover:

TheOREM 1. There exists a bijection $\operatorname{Ext}(A, \mathbb{B}) \rightarrow H^{2}(A, \mathbb{B})$ which is an isomorphism in the category of spiders [6] and which maps the trivial $M$-extension onto the null class.

The bijection is given by associating with each extension a factor system, which then corresponds to a 2-cocycle. An equivalence class of extensions will then correspond to an orbit of 2-cocycles.

4. Functorial properties. The functorial properties we desire are best described by the introduction of the category $C$. The objects of $\mathrm{C}$ are the systems $\beta=\{B, M ; \rho, \Phi\}$ of $\S 1$. A morphism $\tilde{\sigma}:\left\{B_{1}, M_{1} ; \rho_{1}, \Phi_{1}\right\} \rightarrow\left\{B_{2}, M_{2} ; \rho_{2}, \Phi_{2}\right\}$ in $C$ is a pair $\sigma: B_{1} \rightarrow B_{2}, s: M_{1} \rightarrow M_{2}$ of algebra homomorphisms for which $\rho_{2} \circ \sigma=s \circ \rho_{1}$ and $\sigma(b m)$ $=\sigma(b) s(m), \sigma(m b)=s(m) \sigma(b)$, for all $m \in M_{1}, b \in B_{1}$. (We write $b m$ for $b \Phi_{1}(m)$, etc. $)$ 
Every morphism $\tilde{\sigma}$ in $\mathbf{C}$ induces a morphism $\mathfrak{e}^{1}\left(A, \bigotimes_{1}\right) \rightarrow \mathfrak{e}^{1}\left(A, \bigotimes_{2}\right)$ of groupoids, which maps $Z^{1}\left(A, \beta_{1}\right)$ into $Z^{1}\left(A, \beta_{2}\right)$. Under this induced morphism, $(p, \phi) \in \mathfrak{C}^{1}\left(A, \bigotimes_{1}\right)$ is mapped on to $(\sigma p, s \phi) \in \mathfrak{C}^{1}\left(A, \bigotimes_{2}\right)$. Also, $\tilde{\sigma}$ induces a map $Z^{2}\left(A, \beta_{1}\right) \rightarrow Z^{2}\left(A, \beta_{2}\right)$ which sends $\left(\gamma_{1}, \gamma_{2}, \phi\right)$ $\in Z^{2}\left(A, \beta_{1}\right)$ into $\left(\sigma \gamma_{1}, \sigma \gamma_{2}, s \phi\right)$. Since

$$
\sigma \delta_{1}(p, \phi)=\delta_{1}(\sigma p, s \phi), \quad \sigma \delta_{2}(p, \phi)=\delta_{2}(\sigma p, s \phi),
$$

the images of two 2-cocycles in the same orbit of $Z^{2}\left(A, B_{1}\right)$ also lie in the same orbit of $Z^{2}\left(A, \bigotimes_{2}\right)$. Hence $\tilde{\sigma}$ induces a map $\Sigma: H^{2}\left(A, \bigotimes_{1}\right)$ $\rightarrow H^{2}\left(A, \mathrm{~B}_{2}\right)$.

Theorem 2. For a fixed algebra $A, \mathfrak{C}^{1}, Z^{1}, Z^{2}$ and $H^{2}$ are covariant functors with domain $\mathrm{C}$. The codomain of $\mathrm{e}^{1}$ and $\mathrm{Z}^{1}$ is the category of groupoids, and the codomain of $Z^{2}$ and $H^{2}$ is the category of (based) spiders.

5. Exact sequences. A sequence of morphisms in $\boldsymbol{C}$ :

$$
\AA_{1} \stackrel{\tilde{\sigma}_{1}}{\rightarrow} \bigotimes_{2} \stackrel{\tilde{\sigma}_{2}}{\rightarrow} B_{3}, \quad B_{i}=\left\{B_{i}, M_{i}, \rho_{i}, \Phi_{i}\right\}
$$

gives a commutative diagram

$$
\begin{gathered}
0 \rightarrow B_{1} \stackrel{\sigma_{1}}{\rightarrow} B_{2} \stackrel{\sigma_{2}}{\rightarrow} B_{3} \rightarrow 0 \\
\downarrow \rho_{1} \downarrow \rho_{2} \quad \downarrow \rho_{3} \\
M_{1} \stackrel{s_{1}}{\rightarrow} M_{2} \stackrel{s_{2}}{\rightarrow} M_{3} .
\end{gathered}
$$

The sequence (11) will be called a short-exact sequence if

the top row in (12) is an exact sequence of algebras;

$s_{1}$ is an isomorphism of algebras;

$s_{2}$ is an epimorphism of algebras.

The sequence (11) induces the sequence

$$
H^{2}\left(A, \beta_{1}\right) \stackrel{\Sigma_{1}}{\rightarrow} H^{2}\left(A, \beta_{2}\right) \stackrel{\Sigma_{2}}{\rightarrow} H^{2}\left(A, \beta_{3}\right) .
$$

TheOREM 3. If (11) is short-exact, then (14) is exact.

The exactness of (14) is in the sense that, an element of $H^{2}\left(A, B_{2}\right)$ is in the image of $\Sigma_{1}$ if and only if its image under $\Sigma_{2}$ is neutral.

Henceforth we assume we have a short-exact sequence (11). The fibres of the map $\Sigma_{1}$ in (14) can then be described in terms of an action $\diamond$ of $\mathcal{Z}^{1}\left(A, B_{3}\right)$ on $H^{2}\left(A, B_{1}\right)$. Specifically, we take $\left(\gamma_{1}, \gamma_{2}, \phi\right)$ $\in Z^{2}\left(A, \Theta_{1}\right)$ and $(p, \psi) \in Z^{1}\left(A, \Theta_{3}\right)$ such that $\psi=s_{2} s_{1} \phi$. Then lift $(p, \psi)$ 
to $\left(q, s_{1} \phi\right) \in \mathbb{C}^{1}\left(A, B_{2}\right)$ taking $q: A \rightarrow B_{2}$ such that $\sigma_{2} q=p$. Check that $\Delta\left\{\left(q, s_{1} \phi\right) x\left(\sigma_{1} \gamma_{1}, \sigma_{1} \gamma_{2}, s_{1} \phi\right)\right\} \in Z^{2}\left(A, \beta_{2}\right)$ is the image under $\tilde{\sigma}_{1}$ of a (necessarily unique) element $\left(\beta_{1}, \beta_{2}, \chi\right) \in Z^{2}\left(A, \beta_{1}\right)$, where

$$
\chi=s_{1}^{-1}\left(\rho_{2} q+s_{1} \phi\right) .
$$

Check that the class of $\left(\beta_{1}, \beta_{2}, \chi\right)$ is independent of the choice of $q$ and define the action $\diamond$ setting

$$
(p, \psi) \diamond\left[\gamma_{1}, \gamma_{2}, \phi\right]=\left[\beta_{1}, \beta_{2}, \chi\right]
$$

where the brackets indicate the cohomology class of a cocycle. This is a groupoid action, defined iff $\psi=s_{2} s_{1} \phi$.

TheOREM 4. If (11) is short-exact, then the fibres of the map $\Sigma_{1}$ in (14) are the orbits in $H^{2}\left(A, B_{1}\right)$ under the $\diamond$-action of $\mathrm{Z}^{1}\left(A, \Theta_{3}\right.$.)

6. The longer exact sequence. Take $B=\{B, M ; \rho, \Phi\}$ in $C$, and take $\phi: A \rightarrow M$ to be a fixed homomorphism. Denote by $Z_{\phi}^{1}(A, \beta)$ the subset of $Z^{1}(A, \beta)$ of those elements of the form $(p, \phi) . Z_{\phi}^{1}(A, ß)$ is then endowed with a base point $(0, \phi)$ and with a structure of polypus (cf. [1], [4]). In $H^{2}(A, \beta)$, the class of $(0,0, \phi)$ will be called $\phi$-null, and with this as base point, our based set will be denoted by $H_{\phi}^{2}(A, \beta)$.

In a short-exact sequence (11), we can identify $B_{1}$ with its image under $\sigma_{1}$, and $M_{1}, M_{2}$ under the isomorphism $s_{1}$ will be denoted by $M$. Let $M^{\prime}=M_{3}$. Take $\phi: A \rightarrow M$ to be a fixed homomorphism, and put $\phi^{\prime}=s_{2} \phi: A \rightarrow M^{\prime}$. Then

$$
* \rightarrow Z_{\phi}^{1}\left(A, \otimes_{1}\right) \rightarrow Z_{\phi}^{1}\left(A, \AA_{2}\right) \rightarrow Z_{\phi^{\prime}}{ }^{\prime}\left(A, \otimes_{3}\right)
$$

is an exact sequence of based sets and even of polypi [1]. We define $Z_{\phi}^{1}\left(A, B_{3}\right) \rightarrow H_{\phi}^{2}\left(A, B_{1}\right)$ to be the map which sends each element of $Z_{\phi}^{1}\left(A, B_{3}\right)$ onto the result of its action (cf. $\S 5$ ) on the $\phi$-null class of $H_{\phi}^{2}\left(A, \Theta_{1}\right)$. With this definition we have

THeOREM 5. If (11) is short exact, then

$$
\begin{aligned}
* \rightarrow Z_{\phi}^{1}\left(A, B_{1}\right) \rightarrow Z_{\phi}^{1}\left(A, \Theta_{2}\right) & \rightarrow Z_{\phi^{\prime}}^{1}\left(A, \Theta_{3}\right) \rightarrow \\
& \stackrel{\Delta}{\rightarrow} H_{\phi}^{2}\left(A, B_{1}\right) \stackrel{\Sigma_{1}}{\rightarrow} H_{\phi}^{2}\left(A, \bigotimes_{2}\right) \stackrel{\Sigma_{2}}{\rightarrow} H_{\phi^{\prime}}^{2}\left(A, \bigotimes_{3}\right)
\end{aligned}
$$

is exact in the following sense: an element of $Z_{\phi}^{1}\left(A, \beta_{3}\right)$ is in the image of the preceding map if and only if its image under the following map is neutral; an element of $H_{\phi}^{2}\left(A, \Theta_{1}\right)$ (resp. $H_{\phi}^{2}\left(A, \Theta_{2}\right)$ ) lies in the image of the preceding map if and only if its image under the following map is $\phi$-null (resp. neutral). 
It is also possible to characterize the fibres of all maps in (15) and the tricks involved should be incorporated in the notion of exactness. The most difficult fibres are those of $\Sigma_{2}$ and, in the case of group extensions, a method has just been described in [6]: it involves a refinement of the spider structure on $H^{2}(A, B)$.

\section{REFERENCES}

1. P. Dedecker, Le foncteur Hom non abélien. Notion de poulpe, C. R. Acad. Sci. Paris 257 (1963), 2384-2387; Applications de la notion de poulpe, 258 (1964), 11171120.

2. - - Les foncteurs Ext, $H_{\pi}^{2}$ et $H_{\pi}$, C. R. Acad. Sci. Paris 258 (1964), 48914894. Premier dérivé du foncteur Hom non abélien, 259 (1964), 2054-2057.

3. - Cohomologie non abêlienne, (Seminar notes), Faculté des Sciences de Lille (1965).

4. A. S.-T. Lue, Non abelian cohomology of associative algebras, Quart. J. Math. (Oxford) (to appear).

5. S. MacLane, Extensions and obstructions for rings, Illinois J. Math. 2 (1958), 316-345.

6. P. Dedecker and A. Frei, Les relations d'équivalence des morphismes de la suite exacte de cohomologie non abélienne, C. R. Acad. Sci. Paris 262 (1966); Généralisation de la suite exacte de cohomologie non abélienne 262 (1966).

Instituts de Mathématiques, Universités de Lille et DE LoUvain KING'S COLLEGE, LONDON 